\title{
Análise da Transferência de Calor de um Redutor de Velocidade
}

\section{Analysis of Heat Transfer of a Speed Reducer}

\author{
Vanessa Lourenço Pierini ${ }^{1}$; Fernando Henrique Milanese²; Carolina Resmini Melo ${ }^{3}$; \\ Aline Resmini Melo ${ }^{4}$
}

\section{Resumo}

O presente trabalho tem como objetivo analisar a transferência de calor e a eficiência da aleta em um redutor de velocidade Coroa/Sem Fim. O material do redutor é o alumínio e a redução é de 15:1. Foi utilizado um motor de $1 / 2 \mathrm{HP}$ acoplado ao redutor e realizados testes com e sem carga. Os experimentos foram realizados em uma bancada de teste de freio eletromagnético (Freio de Foucault) em convecção natural. Para a realização das medidas de temperatura, utilizou-se o aparelho HBM QUANTUM MX-840A e um termopar de contato tipo $\mathrm{K}$. O cálculo do coeficiente de convecção foi realizado pelo modelo matemático teórico e experimental, sendo que o método experimental prevaleceu no trabalho, pois o método teórico possui algumas restrições. A eficiência da aleta foi calculada pelo método unidimensional. Para uma transferência de calor mais eficiente, foi proposta a alteração da geometria da aleta e realizados testes em convecção forçada, com isso, obteve-se uma redução da temperatura da superfície. Os valores da eficiência da aleta obtidos foram todos próximos devido às pequenas faixas de temperaturas que ocorreram no experimento. Para todos os testes foi utilizado um termovisor para a obtenção de imagens térmicas e comparação dos resultados.

Palavras-chave: Aleta freio eletromagnético. Redutor. Transferência de calor.

\begin{abstract}
This study aims to analyze the heat transfer fin efficiency and a speed reducer Crown / Endless. The material is aluminum reducer and the reduction is $15: 1$. We used a $1 / 2$ HP motor and gearbox coupled to tests conducted with and without load. The experiments were performed on a test bench electromagnetic brake (brake Foucault) in natural convection. To perform the temperature measurements, we used the device HBM QUANTUM MX-840A and a contact thermocouple type K. The calculation of the convection coefficient was performed by experimental and theoretical mathematical model, and the experimental method prevailed at work, because the theoretical method has some restrictions. The fin efficiency was calculated by one-dimensional. For a more efficient heat transfer, it was proposed to change the geometry of the flap and conducted tests on forced convection, thus, there was obtained a reduction of the surface temperature. The values of fin efficiency obtained were all close due to small temperature ranges that occurred in the experiment. For all testing a thermal imager was used to obtain thermal images and comparing the results.
\end{abstract}

Key words: Extended surface. Electromagnetic brake. Reducer. Heat transfer.

\footnotetext{
Aluna de Graduação do Departamento de Engenharia Mecânica, Faculdade Satc; eng.vanessapierini@yahoo.com

Professor Titular da Universidade Federal de Santa Catarina - UFSC; fernando.milanez@reitoria.ufsc.br

3 Docente do Departamento de Engenharia Química da Faculdade Satc; carolina.melo@satc.edu.br

4 Coordenadora e Docente do Curso de Engenharia Química da Faculdade Satc; aline.melo@satc.edu.br
} 


\section{Lista de Símbolos}

\begin{tabular}{|c|c|c|}
\hline$a$ & {$\left[\mathrm{~m}^{2} / \mathrm{s}\right]$} & Difusividade térmica \\
\hline$a$ & {$\left[\mathrm{~m}^{2} / \mathrm{s}\right]$} & Difusividade térmica (superficie horizontal) \\
\hline$a$ & {$\left[\mathrm{~m}^{2} / \mathrm{s}\right]$} & Difusividade térmica (placa vertical) \\
\hline$\beta$ & {$\left[\mathrm{K}^{-1}\right]$} & Coeficiente de expansão térmica \\
\hline$\beta_{h}$ & {$\left[\mathrm{~K}^{-1}\right]$} & $\begin{array}{l}\text { Coeficiente de expansão térmica (superficie } \\
\text { horizontal) }\end{array}$ \\
\hline & {$\left[\mathrm{K}^{-1}\right]$} & Coeficiente de expansão térmica (placa vertical) \\
\hline
\end{tabular}

$\boldsymbol{\Delta} T[\mathrm{~K}] \quad$ Variação de temperatura

$\boldsymbol{\Delta} T_{\boldsymbol{h}} \quad[\mathrm{K}] \quad$ Variação de temperatura (superficie horizontal)

$\Delta T_{\text {smt }}[\mathrm{K}] \quad$ Variação de temperatura média da superficie total

$\boldsymbol{\Delta} T_{v} \quad[\mathrm{~K}] \quad$ Variação de temperatura (placa vertical)

$\Delta v \quad[\mathrm{~W}] \quad$ Variação da energia interna

$\eta_{a} \quad[---] \quad$ Eficiência da aleta

$\eta_{\mathrm{m}} \quad$ [---] Eficiência do motor

v $\quad\left[\mathrm{m}^{2} / \mathrm{s}\right] \quad$ Viscosidade cinemática

$\begin{array}{lll}v_{\boldsymbol{h}} & {\left[\mathrm{m}^{2} / \mathrm{s}\right] \quad \text { Viscosidade cinemática (superficie horizontal) }}\end{array}$

$v_{v} \quad\left[\mathrm{~m}^{2} / \mathrm{s}\right] \quad$ Viscosidade cinemática (placa vertical)

Ac $\quad\left[\mathrm{m}^{2}\right] \quad$ Área da seção transversal

Af $\left[\mathrm{m}^{2}\right] \quad$ Área da superficie total da aleta

$A_{0} \quad\left[\mathrm{~m}^{2}\right] \quad$ Área da superficie primária (sem aletas)

$A_{s p} \quad\left[\mathrm{~m}^{2}\right] \quad$ Área da superficie da placa

$A_{s t} \quad\left[\mathrm{~m}^{2}\right] \quad$ Área total da superficie

$\cos \theta \quad[---] \quad$ Fator de potência

d $\quad[\mathrm{m}] \quad$ Braço de momento

F [N] Força de momento

$g \quad\left[\mathrm{~m} / \mathrm{s}^{2}\right]$ Aceleração da gravidade

$\overline{\boldsymbol{h}} \quad\left[\mathrm{W} / \mathrm{m}^{2} . \mathrm{K}\right] \quad$ Coeficiente de transferência de calor por convecção médio

$\overline{\boldsymbol{h}_{\boldsymbol{h}}} \quad\left[\mathrm{W} / \mathrm{m}^{2} . \mathrm{K}\right] \quad$ Coeficiente de transferência de calor por convecção médio (superficie horizontal)

$\overline{\boldsymbol{h}_{\mathrm{v}}} \quad\left[\mathrm{W} / \mathrm{m}^{2} . \mathrm{K}\right] \quad$ Coeficiente de transferência de calor por convecção médio (placa vertical) 


\begin{tabular}{|c|c|c|}
\hline$l_{f}$ & {$[\mathrm{~A}]$} & Corrente do freio \\
\hline$l_{-}$ & {$[\mathrm{A}]$} & Corrente do motor \\
\hline$k_{a}$ & {$[\mathrm{~W} / \mathrm{m} \cdot \mathrm{K}]$} & Condutividade térmica do alumínio \\
\hline$k_{z}$ & {$[\mathrm{~W} / \mathrm{m} \cdot \mathrm{K}]$} & Condutividade térmica do ar \\
\hline$k_{z} \boldsymbol{h}$ & {$[\mathrm{W} / \mathrm{m} \cdot \mathrm{K}]$} & Condutividade térmica do ar (superficie horizontal) \\
\hline & {$[\mathrm{W} / \mathrm{m} \cdot \mathrm{K}]$} & Condutividade térmica do ar (placa vertical) \\
\hline 2 & {$[\mathrm{~m}]$} & Comprimento característico da geometria \\
\hline & {$[\mathrm{m}]$} & Comprimento da aleta \\
\hline & {$[\mathrm{m}]$} & Comprimento corrigido \\
\hline & {$[\mathrm{m}]$} & $\begin{array}{l}\text { Comprimento característico da geometria (superficie } \\
\text { horizontal) }\end{array}$ \\
\hline & {$[\mathrm{m}]$} & Comprimento da placa \\
\hline & {$[\mathrm{m}]$} & $\begin{array}{l}\text { Comprimento característico da geometria (placa } \\
\text { vertical) }\end{array}$ \\
\hline$m$ & {$\left[\mathrm{~m}^{-2}\right]$} & Constante da aleta \\
\hline$M t$ & {$[\mathrm{~N} . \mathrm{m}]$} & Torque do redutor \\
\hline$n_{s}$ & {$[\mathrm{rpm}]$} & Rotação de saida \\
\hline$\overline{N u}_{2}$ & {$[---]$} & Número de Nusselt \\
\hline$\overline{N u}_{2 \mathbf{h}}$ & {$[---]$} & Número de Nusselt (superficie horizontal) \\
\hline$\overline{N u}_{t=}$ & {$[---]$} & Número de Nusselt (placa vertical) \\
\hline$P_{f}$ & {$[\mathrm{~W}]$} & Potência do freio \\
\hline$P_{L}$ & {$[\mathrm{~m}]$} & Perimetro da aleta \\
\hline$=$ & {$[\mathrm{W}]$} & Potência do motor \\
\hline & {$[\mathrm{W}]$} & Potência mecânica do motor \\
\hline & {$[\mathrm{m}]$} & Perímetro da placa \\
\hline & {$[---]$} & Número de Prandtl. \\
\hline$-\boldsymbol{h}$ & {$[---]$} & Número de Prandtl (superficie horizontal) \\
\hline & {$[\%]$} & Potência fornecida em relação a nominal \\
\hline P & {$[---]$} & Número de Prandtl (placa vertical) \\
\hline 8 & [W] & Energia que entra \\
\hline$\theta$ & [W] & Taxa de transferência de calor por convecção \\
\hline Q & {$[\mathrm{W}]$} & Calor real da aleta \\
\hline & {$[\mathrm{W}]$} & Calor ideal da aleta \\
\hline
\end{tabular}




\begin{tabular}{|c|c|c|}
\hline$R o_{2}$ & {$[---]$} & Número de Rayleigh \\
\hline$R a_{L h}$ & {$[---]$} & Número de Rayleigh (superficie horizontal) \\
\hline$R a_{L=}$ & {$[---]$} & Número de Rayleigh (placa vertical) \\
\hline$\tau$ & {$[\mathrm{N} . \mathrm{m}]$} & Torque do dinamômetro \\
\hline : & {$[\mathrm{m}]$} & Espessura da aleta \\
\hline$\tau_{f}$ & {$[\mathrm{~K}]$} & Temperatura de filme \\
\hline$\tau_{f \boldsymbol{h}}$ & {$[\mathrm{K}]$} & Temperatura de filme (superficie horizontal) \\
\hline$\tau_{f *}$ & {$[\mathrm{~K}]$} & Temperatura de filme (placa vertical) \\
\hline$\tau_{m a t}$ & {$[\mathrm{~K}]$} & Temperatura média da superficie total \\
\hline$\tau$ & {$[\mathrm{K}]$} & Temperatura da superficie \\
\hline$\tau_{\boldsymbol{s}} \boldsymbol{h}$ & {$[\mathrm{K}]$} & Temperatura da superficie (superficie horizontal) \\
\hline$\tau_{z}$ & {$[\mathrm{~K}]$} & Temperatura da superficie (placa vertical) \\
\hline$\tau_{\infty}$ & {$[\mathrm{K}]$} & Temperatura ambiente \\
\hline v & {$[\mathrm{V}]$} & Tensão medida fase/fase \\
\hline V & {$[\mathrm{V}]$} & Tensão medida fase/neutro \\
\hline$w$ & {$[\mathrm{~W}]$} & Energia que sai \\
\hline$w_{a}$ & {$[\mathrm{~m}]$} & Largura da aleta \\
\hline$w_{v}$ & {$[\mathrm{~m}]$} & Largura da placa \\
\hline
\end{tabular}

\section{Introdução}

Os redutores Coroa/Sem Fim possuem uma eficiência de trabalho em torno de $85 \%$. Essa perda se dá devido ao atrito que ocorre entre a coroa e o sem fim, nos rolamentos e ao movimento do óleo dentro do redutor. Este trabalho é focado na transferência de calor do redutor e na eficiência de transmissão da aleta que, de acordo com Kreith e Bohn (2003), são largamente utilizadas para aumentar a taxa de transferência de calor a partir de uma parede.

$\mathrm{O}$ estudo da transferência de calor em um redutor de velocidade é muito importante, pois o mesmo pode ser aplicado em diversos ambientes, com temperaturas diferentes, portanto, deve-se fazer um controle para que não ocorra um aumento da mesma. Quando o redutor trabalha a uma temperatura acima da normal, os vedadores podem ficar ressecados perdendo suas características de retenção, facilitando o aparecimento de vazamentos indesejáveis. O óleo, por sua vez, em excesso de temperatura, perde suas qualidades lubrificantes, que em casos extremos pode diminuir a vida útil dos rolamentos e do próprio redutor.

Devido a tais problemas que podem ser ocasionados em função do aquecimento, foram realizados testes em um redutor de alumínio, com e sem carga, para analisar a transferência de calor e verificar a eficiência da aleta. Após avaliar o método atual e obter os resultados, foram propostas modificações para aumentar a transferência de calor.

A taxa de transferência de calor pode ser elevada aumentando-se a área da superfície através da qual ocorre a convecção. Isso pode ser feito com o emprego de aletas que se estendem a partir da 
parede em direção ao interior do fluido adjacente (INCROPERA, DEWITT, 2003).

\section{Revisão Bibliográfica}

É apresentada uma revisão bibliográfica a respeito de sistemas térmicos e áreas afins, como transferência de calor (condução, convecção natural e forçada, superfícies estendidas), redutor (princípio de funcionamento e materiais) e freio eletromagnético (Freio de Foucault).

\section{Redutor}

O redutor é uma solução de engenharia cujo objetivo é facilitar a movimentação de máquinas, reduzindo a rotação do eixo e aumentando o torque, sendo aplicado em vários segmentos da indústria.

Existem diversos modelos de redutores, o estudado foi o de Coroa/Sem Fim, sendo fabricado pela Pierini Redutores, com coroa de bronze em liga especial e eixo sem fim em aço SAE 1045 de alta resistência (PIERINI, 2007). Os rolamentos são dimensionados para garantir alta durabilidade e os retentores utilizados para assegurar uma completa vedação.

A liga de alumínio usada na fabricação da carcaça, tampas, pé do redutor e flange é Al-Si. As peças são fabricadas em molde de fundição tradicional em areia. Segundo CORRADI (2001), as séries das ligas de alumínio-silício têm excelentes propriedades mecânicas, tais como elevada condutividade térmica e baixo peso específico, boa usinabilidade e resistência à corrosão, muito boa fluidez e melhor aptidão a moldação, principalmente para as composições entre 5 a $14 \%$ de silício.

A lubrificação interna é feita em óleo Super Cilíndrico $600 \mathrm{~W}$, que possui boa adesividade e resistência de película, ótima resistência à oxidação, elevado índice de viscosidade (sofrendo menos variações de viscosidades com as variações de temperaturas), maior proteção das peças contra ferrugem (MOBIL OIL, 1999).

\section{Freio eletromagnético}

De acordo com SILVEIRA E CORREIA (2011), um sistema de freio eletromagnético (Freio de Foucault) por ação de correntes induzidas é capaz de simular uma carga qualquer em um eixo rotacional de um equipamento. Através da aplicação de uma corrente induzida, o sistema disco-bobinas do freio recebe um torque contrário ao causado pela rotação do eixo do equipamento que está sendo utilizado no experimento. Dessa forma dependendo do valor da corrente contínua aplicada ao freio, o equipamento operará no ponto de operação desejada. Bancadas de laboratório equipadas com freio eletromagnético são unidades importantes para ensaios destes testes.

$\mathrm{O}$ dimensionamento do redutor em relação à bancada de testes do Freio de Foucault foi realizado com as fórmulas a seguir.

Para calcular o torque dos dinamômetros, é utilizada a seguinte fórmula (MELCONIAN, 2008):

$$
\mathbf{T}=\mathbf{F} \times \mathbf{d}
$$

Para o dimensionamento do torque do redutor, será usada a seguinte equação (MELCONIAN, 2008):

$$
\mathrm{Mt}=\frac{30 \times \mathrm{P}_{\mathrm{m}}}{\mathrm{n}_{\mathrm{s}} \times \pi}
$$

\section{Primeira lei da termodinâmica}

A primeira lei da termodinâmica afirma que a energia não pode ser criada nem destruída, somente modificada de uma para outra forma (KREITH; BOHN, 2003).

A variação da energia interna $\boldsymbol{\Delta} U$ é calculada com a seguinte equação (INCROPERA; DEWITT, 2003):

$$
U=W-Q
$$


Como o processo é cíclico, os estados finais e iniciais são iguais, $\log \boldsymbol{\Delta} U=0$, então $W=Q$. Todo o calor que sai do sistema será por convecção, ou seja, $Q=q$.

\section{Transferência de calor por condução}

A transferência de calor por condução é o processo em que o calor flui através de um sólido. No modo de condução, o calor é transferido por mecanismo complexo, no qual os átomos interagem em colisões elásticas e inelásticas, a fim de propagar a energia das regiões de temperatura mais alta para as de temperatura mais baixa (KREITH; BOHN, 2003).

A condutividade térmica do material é classificada como uma propriedade de transporte e indica a taxa pela qual a energia é transferida pelo processo de difusão. Ela depende da estrutura física da matéria, atômica e molecular, que, por sua vez, está relacionada ao seu estado da matéria. O fluxo de calor por condução aumenta com o aumento da condutividade térmica (INCROPERA; DEWITT, 2003).

Os testes foram realizados em uma faixa de temperatura cujos valores são baixos, não ultrapassando $350 \mathrm{~K}$, não apresentando variação significativa na condutividade térmica.

\section{Transferência de calor por convecção}

A transferência de calor por convecção ocorre entre um fluido em movimento e uma superfície quando os dois se encontram a diferentes temperaturas (INCROPERA; DEWITT, 2003).

A taxa de transferência de calor $q$ é calculada com a seguinte fórmula (INCROPERA; DEWITT, 2003):

$$
q=\bar{h} \times A_{s t} \times\left(T_{m s t}-T_{c}\right)
$$

Historicamente, o conceito de coeficiente de convecção foi introduzido por Isaac Newton em 1701, como forma de quantificar a relação entre o fluxo de calor e a diferença de temperatura na interface e longe dela. Trata-se, portanto, de uma lei empírica que, para ser utilizada com sucesso em aplicações práticas, requer a determinação experimental prévia do coeficiente de convecção em função de diversas variáveis como, por exemplo, geometrias, propriedades físicas do fluido, condições de escoamento, etc. De fato, pode-se encontrar na literatura técnica um número bastante expressivo de correlações empíricas construídas para as mais diversas situações (BRANDI, 2010).

A convecção livre é originada quando uma força de corpo atua sobre um fluido no qual existem gradientes de massa específica. O efeito líquido é a força de empuxo, que induz corrente de convecção livre (INCROPERA; DEWITT, 2003).

O coeficiente de convecção livre $\overline{\mathbf{h}}$ em escoamento externo é calculado por (KREITH; BOHN, 2003):

$$
\overline{\boldsymbol{h}}=\frac{\overline{N u_{L}} \times K_{g}}{L} \quad\left[\mathrm{~W} / \mathrm{m}^{2} . \mathrm{K}\right]
$$

Para placa vertical, o comprimento $L$ é medido na peça, para superfície horizontal o valor de $L$ é calculado pela seguinte fórmula (KREITH; BOHN, 2003):

$$
L \equiv \frac{A_{s p}}{P_{p}}
$$

Para placa plana vertical, o número de Nusselt $\overline{N u_{L}}$ é calculado pela equação a seguir (INCROPERA; DEWITT, 2003):

$$
\overline{N u_{L}}=\left\{0,825+\frac{0,387 \times R a_{L}^{\frac{1}{6}}}{\left[1+\left(\frac{0,492}{P r}\right)^{\frac{9}{16}}\right]^{\frac{8}{27}}}\right\}^{2} \quad[---]
$$


Para superfície horizontal da placa aquecida, o número de Nusselt $\overline{N u_{L}}$ é calculado da seguinte forma (KREITH; BOHN, 2003):

(8) $\overline{N u}_{L}=0,54 \times R a_{L}^{\frac{1}{4}}\left(10^{5} \leq R a_{L} \leq 10^{7}\right)[---]$

O número de Rayleigh $R a_{L}$, para convecção livre em escoamento externo, é calculado pela equação (INCROPERA; DEWITT, 2003):

$$
R a_{L}=\frac{g \times \beta \times\left(T_{s}-T_{\infty}\right) \times L^{3}}{v \times \alpha} \quad[---]
$$

Todas as propriedades são avaliadas na temperatura de filme $T_{f}$, que é calculada por (INCROPERA; DEWITT, 2003):

$$
T_{f}=\frac{T_{s}+T_{\infty}}{2}
$$

O coeficiente de expansão térmico volumétrico é uma propriedade termodinâmica do fluido que fornece uma medida da quantidade, na qual a massa específica varia em resposta a uma variação na temperatura a uma pressão constante (INCROPERA; DEWITT, 2003).

O coeficiente de expansão para um gás ideal $\beta$ é obtido da seguinte forma (INCROPERA; DEWITT, 2003):

$$
\beta=\frac{1}{T_{f}}
$$

A convecção forçada ocorre quando o fluxo térmico é influenciado por meios externos, tais como: um ventilador, uma bomba, ventoinha ou ventos atmosféricos (INCROPERA; DEWITT, 2003).

\section{Transferência de calor total da superfície aletada}

A realização de um novo projeto de aleta, de modo a se obter um novo $\boldsymbol{\Delta} T$, é calculada pela seguinte fórmula (WELTY et al., 2001):

(12) $q=\left(A_{0} \times \overline{\boldsymbol{h}} \times \mathbf{\Delta} T\right)+\left(\eta_{a} \times \overline{\boldsymbol{h}} \times \mathbf{\Delta} T \times A_{f}\right)[\mathrm{W}]$

\section{Eficiência da aleta}

Segundo Huang e Shah (1992), aletas são essencialmente usadas para aumentar a área da superfície e, consequentemente, aumentar a taxa de transferência de calor.

O potencial motriz máximo para convecção é a diferença de temperatura entre a base e o fluido. A taxa máxima na qual uma aleta poderia dissipar energia é a taxa que existiria se toda a superfície da aleta estivesse na temperatura da base. Uma definição lógica da eficiência da aleta é (INCROPERA; DEWITT, 2003):

$$
\eta_{a}=\frac{q_{a}}{q_{\max }}
$$

Conforme HUANG E SHAH (1992), a avaliação da eficiência da aleta é feita baseada na idealização da análise unidimensional, espessura uniforme da aleta fina, condutividade térmica uniforme da aleta, coeficiente uniforme de transferência de calor, temperatura ambiente uniforme, e sem diminuição da temperatura da base da aleta. Se um desses itens não for cumprido, o valor da eficiência ideal unidimensional da aleta diminuirá, mas terá relativamente um pequeno efeito, se a eficiência da aleta estiver acima de $80 \%$.

Neste estudo, a fórmula que será usada para calcular a eficiência da aleta é para um modelo unidimensional, pois nesse experimento todas as avaliações baseadas por HUANG E SHAH (1992) variam de modo sensível. 
A eficiência da aleta $\eta_{a}$ é calculada pela seguinte equação (INCROPERA; DEWITT, 2003):

$$
\eta_{a}=\frac{\tanh \left(m \times L_{c}\right)}{m \times L_{c}}
$$

O comprimento corrigido $L_{c}$ é obtido da seguinte forma (INCROPERA; DEWITT, 2003):

$$
L_{c}=L_{a}+\frac{t}{2}
$$

O valor de $m$ é calculado da seguinte forma (KERN, 1979):

$$
m^{2}=\frac{\overline{\boldsymbol{h}} \times P_{L}}{K_{a} \times A_{c}}
$$

Se a aleta for constituída de um material com alta condutividade térmica, o gradiente de temperatura ao longo da mesma, da base à ponta, será baixo e as características de transferência de calor da parede serão bastante melhoradas (KREITH; BOHN, 2003).

\section{Potência}

A potência mecânica do motor $P_{m e}$ foi calculada pela seguinte equação (PANESI, 2006):

$$
P_{m e}=I_{m} \times U \times \sqrt{3} \times \cos \theta \times \eta_{m}
$$

A potência do freio $P_{f}$ é obtida pela seguinte fórmula (CREDER, 2007):

$$
P_{f}=V \times I_{f}
$$

Os valores de fator de potência, rendimento e potência fornecida em relação à nominal, são obtidos pela Figura 1.
Figura 1 - Curva característica em função da potência-Motor elétrico trifásico - rotor de gaiola $-1 / 2 \mathrm{HP}-60 \mathrm{~Hz} 220 / 380 \mathrm{~V}-2 / 1,16 \mathrm{~A}$.

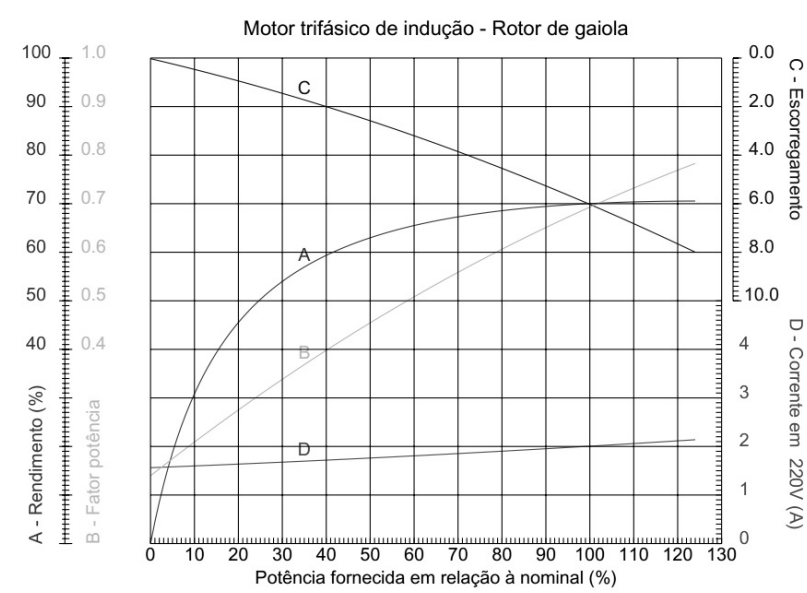

Fonte: WEG, 2012.

\section{Procedimento Metodológico}

O procedimento metodológico foi realizado nas dependências da Faculdade Satc, no Laboratório de Máquinas Elétricas III, para a realização do experimento foram utilizados alguns equipamentos, conforme Tabela1.

Para a realização do experimento, foi necessário dimensionar o redutor conforme as condições dos equipamentos da bancada de teste. Utilizando a Eq. (1), calculou-se o torque máximo do dinamômetro, sendo que este poderia registrar até 11,4 N.m. O modelo elegido para o estudo foi com a redução de 15:1. O motor usado foi de $1 / 2 \mathrm{HP}$, que conforme a Eq. (2) o torque na saída do redutor seria de 31,81 N.m. O conjunto redutor/motor foi escolhido devido às limitações do dinamômetro disponível. A faixa de medição de torque do moto redutor em relação ao dinamômetro seria de $35,84 \%$ da sua capacidade máxima na saída do conjunto. 
Tabela 1 - Lista de materiais utilizados no experimento.

\begin{tabular}{|l|}
\hline \multicolumn{1}{|c|}{ Descrição } \\
\hline Redutor $15: 1$ - Pierini Redutores \\
\hline Motor $1 / 2$ HP - 4 pólos - 220/380V - Weg \\
\hline Analisador de energia - Fluke 43 \\
\hline Multímetro - Fluke 83 III \\
\hline Multímetro - Fluke 115 \\
\hline Alicate amperímetro - Fluke 322 \\
\hline Fonte ajustável de energia (CC) - ETC - 20 \\
\hline Fonte ajustável de energia (CA) - ETC - 20 \\
\hline Termovisor - Fluke ti32 \\
\hline HBM QUANTUM MX-840A \\
\hline Termopar tipo K \\
\hline Acoplamento ACR 03 - Mademil \\
\hline
\end{tabular}

Fonte: Os autores.

$\mathrm{Na}$ realização do experimento, observou-se que as molas contidas nos dinamômetros estavam deformadas, não estando em condições para perfeita medição. Os valores usados para os testes excederam o limite de emprego usual do dinamômetro, devido a este não estar em perfeitas condições. Os valores de torque calculados pela Eq. (2) para o experimento com convecção natural foi de 26,40 N.m , para convecção forçada foi de 28,63 N.m.

Com a definição das equações a serem utilizadas na revisão bibliográfica, realizou-se a coleta de dados do experimento para convecção natural e forçada, conforme descritos na Tabela 2.

No redutor utilizado para os testes, foram feitos 42 furos de diâmetro de $1 \mathrm{~mm}$, podendo assim fixar o termopar para realizar as medições de temperatura. Utilizou-se um termopar de contato tipo $\mathrm{K}$ para as medições, o tempo de contato entre o termopar e a superfície foi em torno de 90 segundos em cada ponto, sendo que em alguns pontos a estabilização levou um tempo maior conforme se observava no Software CatmanEasy-AP ${ }^{\circledR}$. A calibração do aparelho foi feito no Software QuantumX Assistant ${ }^{\circledR}$. Esse processo de medição de temperatura foi realizado nos dois testes, com e sem carga. No teste com carga, o redutor foi acoplado ao motor e o conjunto foi fixado num suporte na bancada de teste, como pode ser visualizado na Figura 2.

\begin{tabular}{|c|c|c|c|c|c|}
\hline \multicolumn{6}{|c|}{ Projeto Atual } \\
\hline \multirow[b]{2}{*}{ Símbolo } & \multirow[b]{2}{*}{ Unidade } & \multicolumn{2}{|c|}{ Convecção natural } & \multicolumn{2}{|c|}{ Conveç̧ão forçada } \\
\hline & & Sem carga & Com carga & Sem carga & Com carga \\
\hline$\tau_{m}$ & $\mathrm{~K}$ & 319,60 & 330,31 & - & $\ldots$ \\
\hline$\tau_{\infty}$ & $\mathrm{K}$ & 298,80 & 298,80 & 297,33 & 297,33 \\
\hline$\Delta \tau_{w}$ & $\mathrm{~K}$ & 20,80 & 31,51 & - & $\ldots$ \\
\hline$t$ & $\mathrm{~m}$ & 0,0063 & 0,0063 & 0,0063 & 0,0063 \\
\hline $\mathrm{La}$ & $\mathrm{m}$ & 0,0627 & 0,0627 & 0,0627 & 0,0627 \\
\hline$W_{a}$ & $\mathrm{~m}$ & 0,0062 & 0,0062 & 0,0062 & 0,0062 \\
\hline$L_{z}$ & $\mathrm{~m}$ & 0,157 & 0,157 & - & $\ldots$ \\
\hline$w_{v}$ & $\mathrm{~m}$ & 0,079 & 0,079 & - & $\ldots$ \\
\hline 2. & $\mathrm{~m}$ & 0,209 & 0,209 & $\ldots$ & $\ldots$ \\
\hline$\tau_{\text {sh }}$ & $\mathrm{K}$ & 321,06 & 334,78 & $\ldots$ & $\ldots$ \\
\hline$\Delta \tau_{\mathbf{h}}$ & $\mathrm{K}$ & 22,26 & 35,98 & - & $\ldots$ \\
\hline$v$ & $\mathrm{v}$ & 220 & 220 & 220 & 220 \\
\hline$l_{m}$ & $\mathrm{~A}$ & 1,60 & 1,92 & 1,69 & 1,96 \\
\hline$v$ & $\mathrm{v}$ & $\ldots$ & 167,13 & $\ldots$ & 162,2 \\
\hline$l_{f}$ & A & $\ldots$ & 0,605 & - & 0,587 \\
\hline$\tau_{m s}$ & $\mathrm{~K}$ & 320,15 & 331,33 & 315,68 & 320,14 \\
\hline$\Delta \tau_{\mathrm{sm}}$ & $\mathrm{K}$ & 21,35 & 32,53 & 18,35 & 22,81 \\
\hline$d$ & $\mathrm{~m}$ & 0,57 & 0,57 & 0,57 & 0,57 \\
\hline$F$ & $\mathrm{~N}$ & 20 & 20 & 20 & 20 \\
\hline
\end{tabular}

Fonte: Os autores.

Figura 2 - Teste do redutor com carga

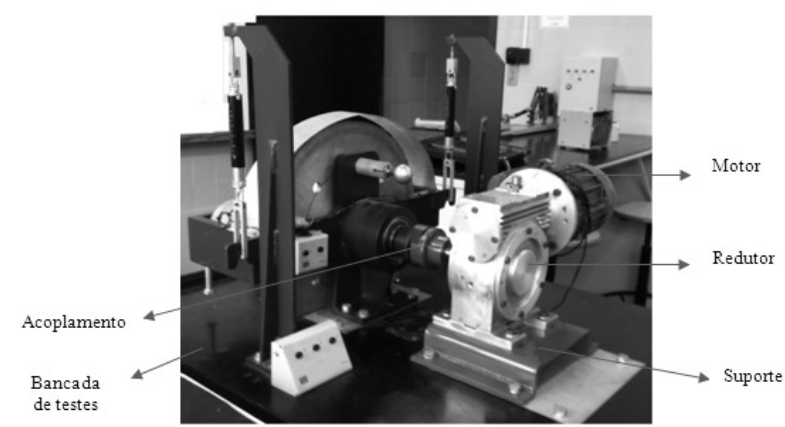

Fonte: Os autores. 
A bancada de testes com o freio de Foucault pode ser visualizado na Figura 3.

Verificou-se o tipo de ligação do motor, tendo em vista que a forma mais adequada de alimentação desse é do tipo triângulo $220 \mathrm{~V}$ fase/fase, devido às limitações da fonte de alimentação. Com o multímetro conectado às fases de entrada do motor, liga-se a fonte ajustável, sendo que seu estado inicial está em $0 \mathrm{~V}$, a partir desse instante aumentou-se a tensão fazendo-a chegar em $220 \mathrm{~V}$ entre suas fases, tendo assim a tensão nominal do motor.

Figura 3 - Freio de Foucault

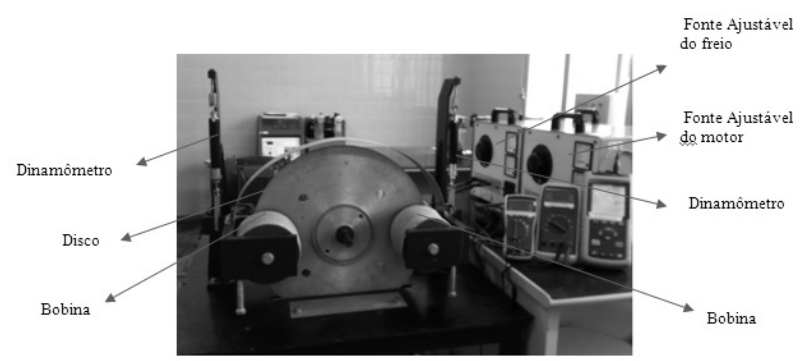

Fonte: Os autores.

Para a aplicação da carga, foi utilizada uma fonte ajustável de energia com saída em corrente contínua, esta por sua vez alimenta as bobinas do freio de Foucault, ficando assim a bancada de teste montada. O eixo do freio eletromagnético é acoplado ao eixo do moto redutor. A carga aplicada ao experimento ficou acionada por duas horas até obter a estabilização da temperatura do redutor, e a mesma pôde ser medida com o aparelho HBM QUANTUM MX-840A, onde este realiza medições a cada 0,02 segundos. Com a temperatura estabilizada, registrou-se imagens do conjunto com o auxílio do termovisor ti32. A termovisão é usada para medir a distribuição de temperatura, podendo assim visualizar as áreas com maior aquecimento e fazer as comparações com os experimentos realizados, o termovisor foi usado em todos os testes.

Para o acionamento do redutor sem carga, usouse uma fonte ajustável de energia em corrente alternada. Foi retirado o acoplamento da ponta do eixo, ficando este sem carga, sendo esperado duas horas para a estabilização da temperatura. A Figura 4 é do redutor sem carga.

Figura 4 - Teste do redutor sem carga.

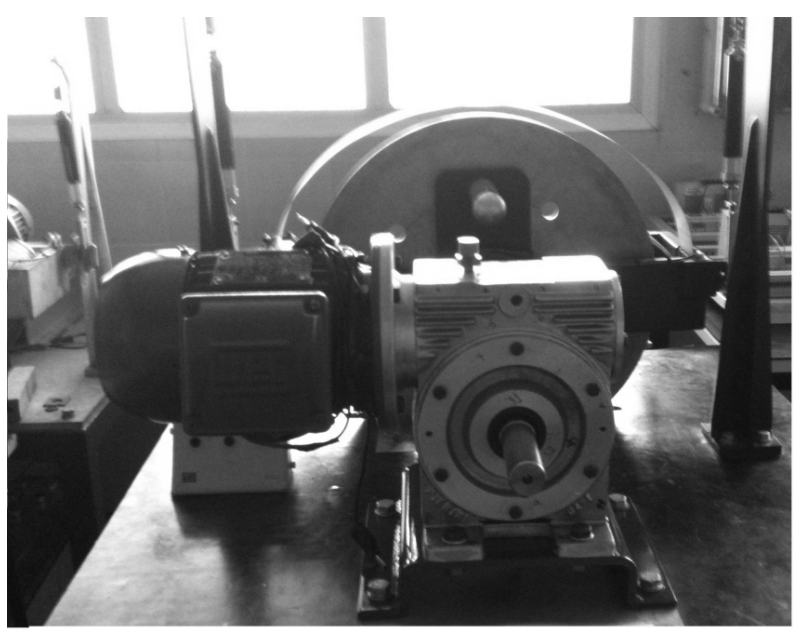

Fonte: Os autores.

Depois de concluídas as análises dos resultados, realizou-se outro teste sendo este com uma ventoinha acoplada ao eixo sem fim, o mesmo teve que ser modificado para fazer este acoplamento. A ventoinha utilizada para a realização deste experimento não foi dimensionada, foi usada uma ventoinha de motor, apenas para verificar a variação de temperatura e as imagens obtidas do termovisor. Os procedimentos dos testes seguiram os mesmos métodos anteriores para o redutor com e sem carga. A Figura 5 apresenta o experimento do redutor com carga e ventoinha. 
Figura 5 - Teste do redutor com carga e com a ventoinha.

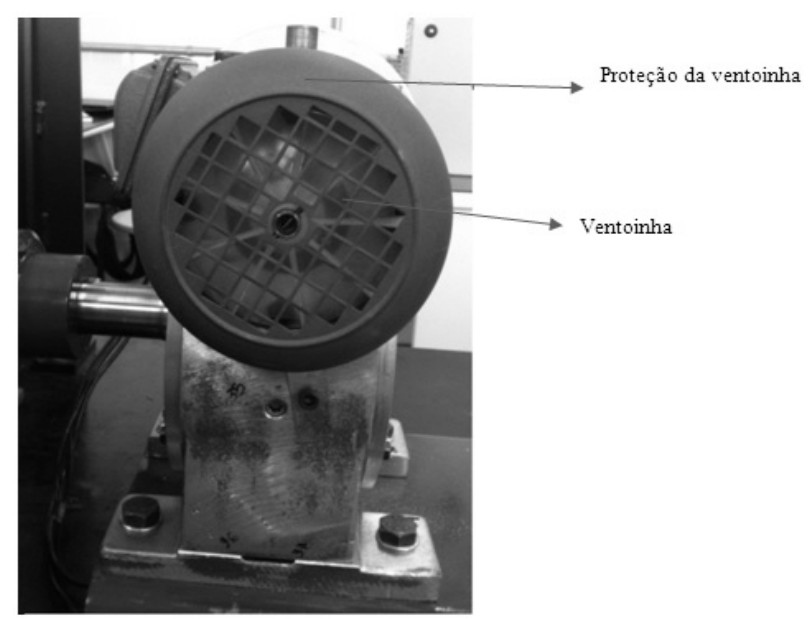

Fonte: Os autores.

\section{Resultado e Análise}

Com os dados coletados no experimento, utilizaram-se as equações já definidas para verificar as condições do projeto atual, foram feitas as análises e descritos os resultados, seguindo a sequência foi apresentada uma condição proposta, a fim de determinar a melhoria no sistema de transferência de calor.

\section{Projeto atual}

A Figura 6 ilustra o projeto atual, a quantidade de aletas, entre outras características. $\mathrm{O}$ modelo foi feito no Software SolidWorks ${ }^{\circledR}$.

Na Tabela 3, pode-se observar os valores obtidos para os cálculos do $\overline{\boldsymbol{h}}$ teórico nas placas verticais e na superfície horizontal.

Os valores de $\alpha_{v}, v_{v}, k_{g v}, P_{r v}, \alpha_{\boldsymbol{h}}, v_{\boldsymbol{h}}, k_{g \boldsymbol{h}}$, $P_{r h}$ foram retirados de tabelas do Incropera e DeWitt (2003), assim como o valor de $g$ que foi retirado da mesma referência.
Figura 6 - Projeto atual das aletas: a-vista frontal, b-vista posterior

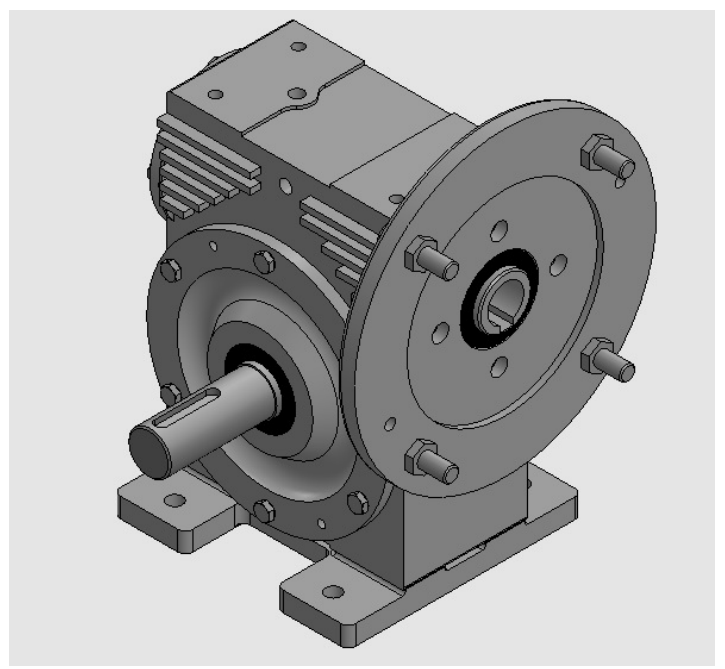

(a)

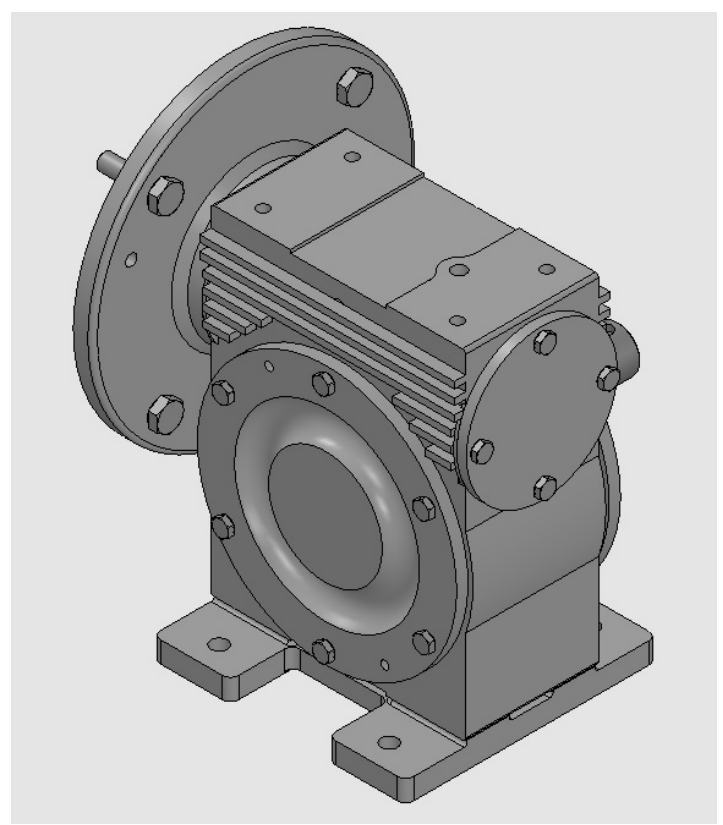

Fonte: Os autores. 
Tabela 3 - Valores calculados utilizando o coeficiente de convecção teórico.

\begin{tabular}{|c|c|c|c|c|}
\hline \multicolumn{5}{|c|}{ Projeto atual - Convecção natural } \\
\hline Símbolo & Unidade & Equaçã̃o & Sem carga & Com carga \\
\hline 9 & $\mathrm{~m} / \mathrm{s}^{2}$ & - & 9,807 & 9,807 \\
\hline$\tau_{p_{v}}$ & $\mathrm{~K}$ & 10 & 309,20 & 314,55 \\
\hline$a_{\text {. }}$ & $\mathrm{m}^{2 / 9}$ & $\ldots$ & $2,39 \mathrm{E}-05$ & $2,47 \mathrm{E}-05$ \\
\hline$y_{v}$ & $\mathrm{~m}^{2 / 9}$ & $\ldots$ & $1,68 \mathrm{E}-05$ & $1,74 \mathrm{E}-05$ \\
\hline$k_{g v}$ & $\mathrm{~W} / \mathrm{m} . \mathrm{K}$ & - & 0,0270 & 0,0274 \\
\hline$P_{w}$ & $\ldots$ & $\ldots$ & 0,7057 & 0,7050 \\
\hline B. & $R^{-1}$ & 11 & 0,00323 & 0,00318 \\
\hline$R a_{b y}$ & $\ldots$ & 9 & $1,51 \times 10^{7}$ & $2,09 \times 10^{7}$ \\
\hline$\overline{N_{z_{b}=}}$ & $\ldots$ & 7 & 35,02 & 38,55 \\
\hline $\bar{h}$ & $\mathrm{~W} / \mathrm{m}^{2} \mathrm{~K}$ & 5 & 4,52 & 5,05 \\
\hline$\tau_{f \mathbf{h}}$ & $\mathrm{K}$ & 10 & 309,93 & 316,79 \\
\hline$a_{h}$ & $\mathrm{~m}^{2} / \mathrm{s}$ & - & $2,40 \mathrm{E}-05$ & $2,50 \mathrm{E}-05$ \\
\hline$y_{h}$ & $\mathrm{~m}^{2 / 9}$ & - & $1,69 \mathrm{E}-05$ & $1,76 \mathrm{E}-05$ \\
\hline$k_{g} h$ & $\mathrm{~W} / \mathrm{m} . \mathrm{K}$ & -- & 0,0270 & 0,0275 \\
\hline$P_{r h}$ & $\ldots$ & $\ldots$ & 0,7056 & 0,7046 \\
\hline$\hat{H}_{h}$ & $R^{-1}$ & 11 & 0,00323 & 0,00316 \\
\hline$P_{5}$ & M & $\ldots$ & 0,47 & 0,47 \\
\hline$A_{25}$ & $\mathrm{~m}^{2}$ & $\ldots$ & 0,0124 & 0,0124 \\
\hline$l_{h}$ & M & 6 & 0,0263 & 0,0263 \\
\hline$R a_{-h}$ & $\ldots$ & 9 & 31571,10 & 45980,29 \\
\hline$\overline{N_{w_{j}, \boldsymbol{h}}}$ & $\ldots$ & 8 & 7,20 & 7,91 \\
\hline $\bar{h}_{h}$ & $\mathrm{~W} / \mathrm{m}^{\mathrm{I}} \mathrm{K}$ & 5 & 7,41 & 8,29 \\
\hline
\end{tabular}

Fonte: Os autores.

Os valores do coeficiente de convecção teórico para essas superfícies foram calculados pela Eq. (5). Para a realização desses cálculos, são consideradas as seguintes hipóteses: as superfícies do redutor são totalmente lisas, o ar permanece parado e a temperatura é a mesma em todos os pontos do redutor.

Foram calculados os coeficientes de convecção teóricos (para as placas verticais e a superfície horizontal) e verificou-se que os mesmos sofreram influência devido às hipóteses consideradas que não ocorrem na prática. Por isso, foi calculado o coeficiente de convecção experimental, para o cálculo utilizou-se a Eq. (4). Os valores obtidos são apresentados Tabela 4, para convecção natural.
Pode-se observar na Tabela 4 que o valor do coeficiente de convecção natural para o teste com carga foi maior. Isso está relacionado à taxa de transferência de calor por convecção que é maior em comparação ao redutor sem carga, junto com este está a potência fornecida em relação à nominal, ou seja, no teste sem carga utilizou-se apenas $15 \%$, enquanto que no teste com carga foram $83 \%$. Ocorreu uma pequena variação da área superficial entre os dois testes, isso ocorreu devido ao fato de um equipamento estar com carga e o outro não. A condutividade térmica do material foi considerada constante em todos os testes.

Tabela 4 - Valores calculados utilizando o coeficiente de convecção experimental.

\begin{tabular}{|c|c|c|c|c|c|c|}
\hline \multicolumn{7}{|c|}{ Valores calculados - Projeto atual } \\
\hline & & & \multicolumn{2}{|c|}{ Convecção natural } & \multicolumn{2}{|c|}{ Conveç̧ão forçada } \\
\hline Símbolo & Unidade & Equação & $\begin{array}{l}\text { Sem } \\
\text { carga }\end{array}$ & $\begin{array}{l}\text { Com } \\
\text { carga }\end{array}$ & $\begin{array}{l}\begin{array}{l}\text { Sem } \\
\text { carga }\end{array} \\
\end{array}$ & Com carga \\
\hline$A_{g}$ & $\mathrm{~m}^{2}$ & --- & $3,98 \times 10^{-4}$ & $3,98 \times 10^{-4}$ & $3,98 \times 10^{-4}$ & $3,98 \times 10^{-4}$ \\
\hline P. & $\mathrm{m}$ & --- & 0,1381 & 0,1381 & 0,1381 & 0,1381 \\
\hline $2=$ & $\mathrm{m}$ & 15 & $9,35 \times 10^{-3}$ & $9,35 \times 10^{-3}$ & $9,35 \times 10^{-3}$ & $9,35 \times 10^{-3}$ \\
\hline $\bar{h}$ & $\mathrm{~W} / \mathrm{m}^{2} \cdot \mathrm{K}$ & 4 & 11,89 & 26,72 & 28,33 & 44,32 \\
\hline$m$ & $\mathrm{~m}^{-2}$ & 16 & 5,03 & 7,53 & 7,75 & 9,69 \\
\hline$r_{a}$ & $\ldots$ & 14 & 0,999265 & 0,998353 & 0,998254 & 0,997271 \\
\hline$q$ & W & 3 & 61,09 & 212,36 & 123,96 & 245,24 \\
\hline$k_{a}$ & W/m.K & --- & 164 & 164 & 164 & 164 \\
\hline$A_{2 t}$ & $\mathrm{~m}^{2}$ & --- & 0,2402 & 0,2444 & 0,2385 & 0,2426 \\
\hline $\cos \theta$ & $\ldots$ & $\ldots$ & 0,25 & 0,62 & 0,35 & 0,65 \\
\hline$\eta_{2}$ & $\ldots$ & --- & 0,4 & 0,69 & 0,55 & 0,7 \\
\hline$P_{m}$ & $\%$ & --- & 15 & 83 & 31 & 90 \\
\hline$P_{m}$ & W & 17 & 61,09 & 313,53 & 123,96 & 340,40 \\
\hline$P_{f}$ & W & 18 & $\ldots$ & 101,17 & $\ldots$ & 95,16 \\
\hline e & W & 3 & 61,09 & 212,36 & 123,96 & 245,24 \\
\hline
\end{tabular}

Fonte: Os autores.

A eficiência da aleta mostrou um valor próximo para ambos os testes. Isso significa que a temperatura da ponta da aleta está muito próxima à temperatura da base da aleta.

A Figura 7 e a Figura 8, são imagens obtidas pelo termovisor em ambos os testes. 
Figura 7 - Imagens do termovisor do teste com carga (convecção natural)

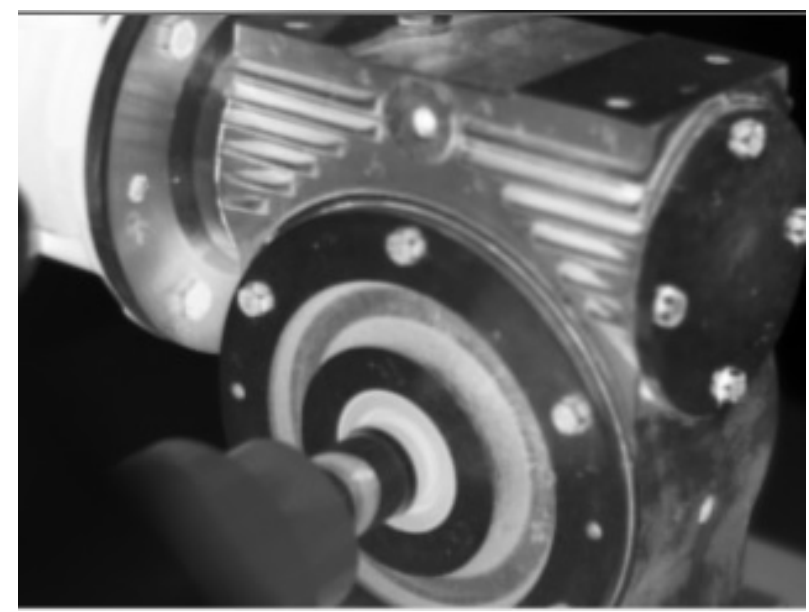

Fonte: Os autores.

É possível observar que não ocorreu uma variação significativa nas imagens com e sem carga. Isso acontece pois o torque do redutor é muito baixo, não ocorrendo uma variação de temperatura muito grande entre os testes, deve-se considerar também a reflexão do alumínio nas imagens. As partes com maior temperatura são aquelas onde se localizam os rolamentos e as engrenagens (Coroa/Sem Fim), onde estão sendo gerados os fluxos térmicos devido à aplicação de carga.

Figura 8 - Imagens do termovisor do teste sem carga(convecçãonatural).

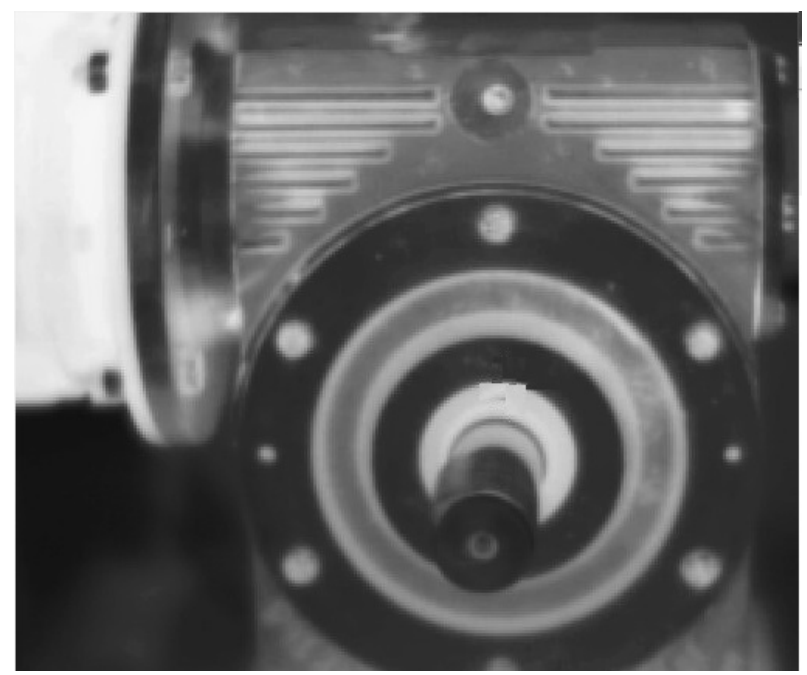

Fonte: Os autores.

\section{Novo projeto}

Depois de feita a análise, foi sugerido um novo modelo, fazendo-se ainda o uso de aletas retangulares. O modelo foi feito no Software SolidWorks ${ }^{\circledR}$. A Figura 9 representa o novo modelo.

Figura 9 - Novo projeto de aletas: a-vista frontal, b-vista posterior.

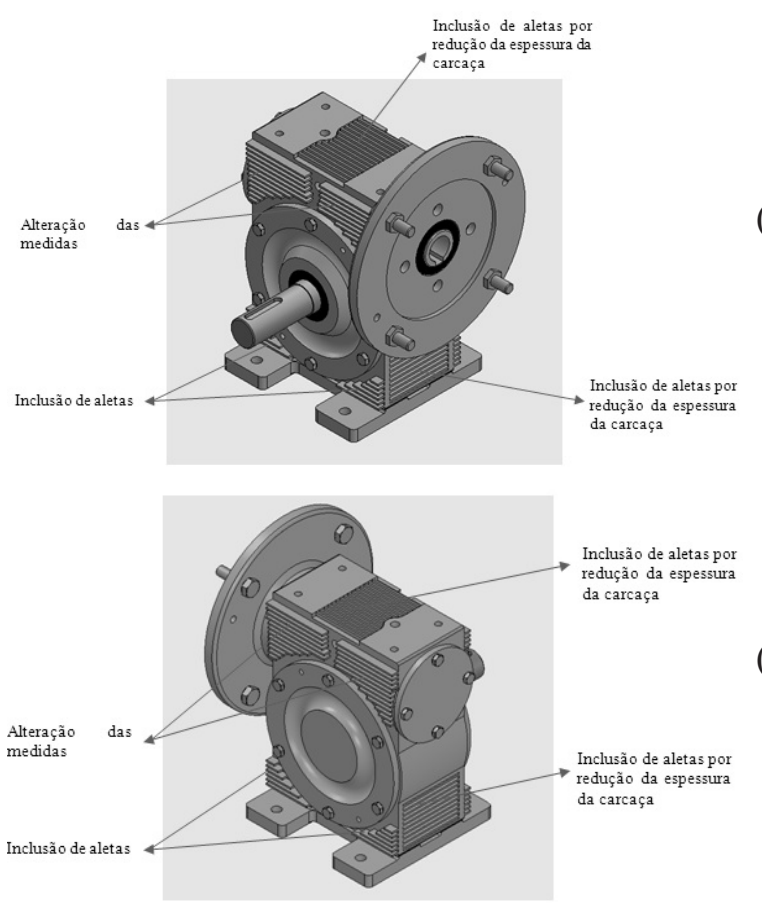

Fonte: Os autores.

Foram acrescentadas aletas em outras posições do redutor que não possuíam, principalmente nos pontos de geração de calor onde estão os rolamentos e o engrenamento da Coroa com o Sem Fim, visando o aumento da área de troca de calor. Foram alterados o comprimento, largura e a espessura da aleta. Sugeriu-se o redimensionamento por redução de espessura da carcaça em algumas partes, a fim de aumentar a eficiência de troca de calor.

O novo projeto calculado pela Eq. (12) apresentou valores diferentes das áreas referentes ao projeto atual. $\mathrm{O}$ valor do coeficiente de convecção foi considerado o mesmo do projeto atual, pois este 
só teria variação significativa se fosse para valores muito diferentes de temperatura e área.

Os valores obtidos de $\boldsymbol{\Delta} T$ e as novas dimensões para esse novo projeto podem ser visualizados na Tabela 5, para convecção natural.

Tabela 5 - Valores calculados no novo projeto.

\begin{tabular}{|c|c|c|c|c|c|c|}
\hline \multicolumn{7}{|c|}{ Novo projeto } \\
\hline \multirow[b]{2}{*}{ Símbolo } & \multirow[b]{2}{*}{ Unidade } & \multirow[b]{2}{*}{ Equação } & \multicolumn{2}{|c|}{ Convecção natural } & \multicolumn{2}{|c|}{ Convecção forçada } \\
\hline & & & Sem carga & Com carga & Sem carga & Com carga \\
\hline$A_{g}$ & $\mathrm{~m}^{2}$ & -- & 0,00014 & 0,00014 & 0,00014 & 0,00014 \\
\hline$P_{L}$ & $\mathrm{~m}$ & -- & 0,144 & 0,144 & 0,144 & 0,144 \\
\hline Le & $\mathrm{m}$ & -- & 0,009 & 0,009 & 0,009 & 0,009 \\
\hline $\bar{h}$ & $\mathrm{~W} / \mathrm{m}^{2} \cdot \mathrm{K}$ & 4 & 11,89 & 26,72 & 28,33 & 44,32 \\
\hline$m$ & $\mathrm{~m}^{-2}$ & 16 & 8,64 & 12,94 & 13,33 & 16,67 \\
\hline$\eta_{a}$ & $\ldots$ & 14 & 0,997988 & 0,99550 & 0,995231 & 0,992562 \\
\hline 9 & $\mathrm{~W}$ & 3 & 61,09 & 212,36 & 123,96 & 245,24 \\
\hline$k_{a}$ & $\mathrm{~W} / \mathrm{m} \cdot \mathrm{K}$ & --- & 164 & 164 & 164 & 164 \\
\hline$A_{f}$ & $\mathrm{~m}^{2}$ & --- & 0,0792 & 0,0792 & 0,0792 & 0,0792 \\
\hline$A_{0}$ & $\mathrm{~m}^{2}$ & --- & 0,2176 & 0,2218 & 0,2159 & 0,22 \\
\hline$W_{a}$ & $\mathrm{~m}$ & --- & 0,07 & 0,07 & 0,07 & 0,07 \\
\hline$L_{a}$ & $\mathrm{~m}$ & --- & 0,008 & 0,008 & 0,008 & 0,008 \\
\hline t & $\mathrm{m}$ & --- & 0,002 & 0,002 & 0,002 & 0,002 \\
\hline$\Delta \tau$ & $\mathrm{K}$ & 12 & 17,29 & 26,44 & 14,85 & 18,53 \\
\hline
\end{tabular}

Fonte: Os autores.

Com o aumento da área superficial de transferência de calor, a temperatura diminui em ambos os testes com e sem carga, melhorando assim a transferência de calor no redutor. Com a diminuição do $\boldsymbol{\Delta} T$ (entre a superfície e o ambiente), consequentemente ocorre também a diminuição da temperatura interna do óleo.

A eficiência da aleta foi calculada pela Eq. (14), apresentando valores altos, e como pode ser observado na Tabela 5, para convecção natural foi obtido valores próximos do projeto atual.

\section{Convecção forçada}

Com o intuito de obter novos valores de $\boldsymbol{\Delta} T$ e coeficiente de convecção, foi realizado um experimento em convecção forçada conforme a Figura 5.

Os resultados obtidos neste experimento foram para o projeto atual e para o novo projeto de aletas, conforme podem ser observados os valores na Tabela 4 e Tabela 5 (para convecção forçada).

No projeto atual, ocorreu uma variação da área, pois para colocar a ventoinha foi retirada uma peça do redutor. Para o redutor sem carga, a potência relativa nominal foi de $31 \%$, ou seja, comparado com a convecção natural ocorreu esta diferença devido ao uso da ventoinha de tamanho desproporcional a carcaça do redutor, ocorrendo o consumo de potência do motor pela ventoinha. Para o teste com carga, usou-se $90 \%$, sendo este próximo à convecção natural.

Com o uso da ventoinha, o $\boldsymbol{\Delta} T$ diminuiu, conforme pode ser observado na Tabela 2 para convecção forçada, isso ocorre devido à diminuição da temperatura da superfície com o ambiente, que consequentemente ocorre devido ao uso da ventoinha, aumentando ainda mais a taxa de transferência de calor por convecção.

A eficiência da aleta não sofreu alteração significativa.

Na Figura 10 e Figura 11 podem ser observadas as imagens obtidas pelo termovisor em ambos os testes. 
Figura 10 - Imagens do termovisor do teste com carga (convecção forçada).

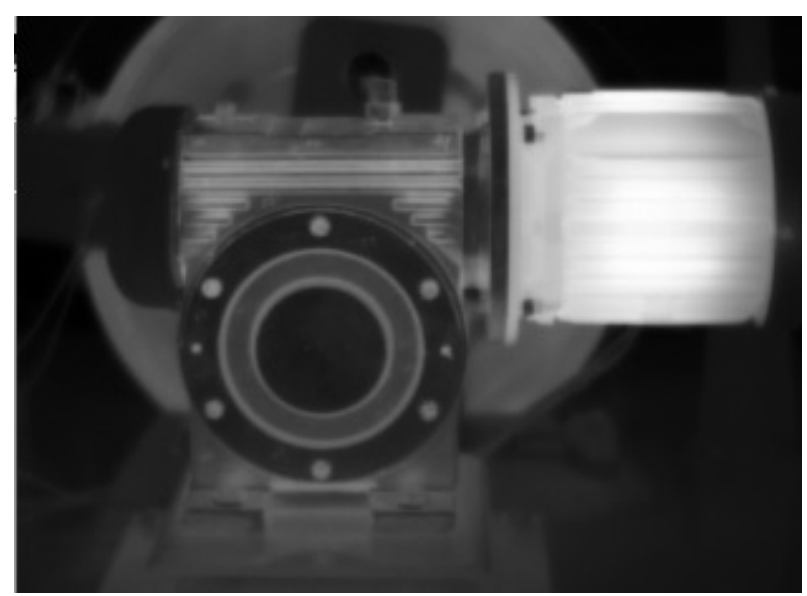

Fonte: Os autores.

Figura 11 - Imagens do termovisor do teste sem carga (convecção forçada)

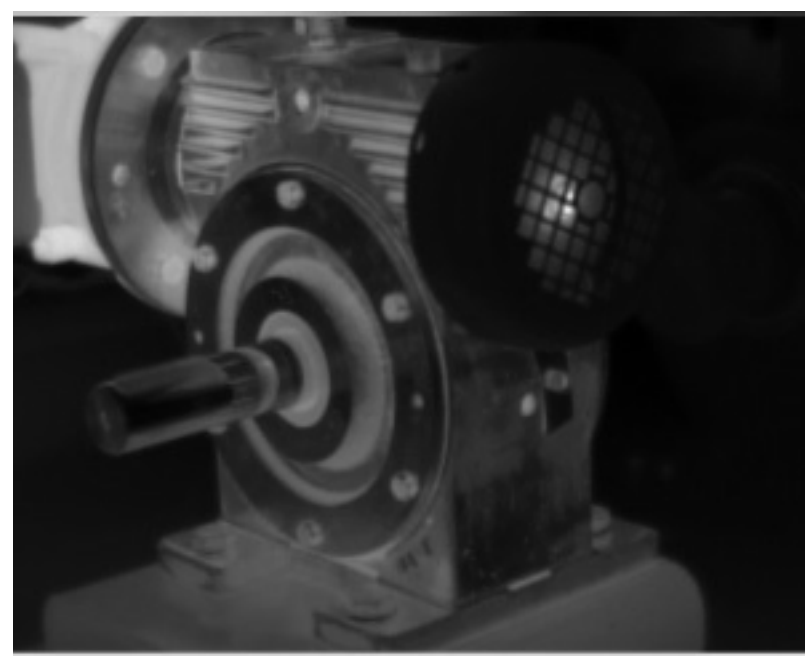

Fonte: Os autores.

Comparando a Figura 10 e Figura 11, obtidas em convecção forçada com a Figura 7 e Figura 8 em convecção natural, pode-se observar que a ventoinha melhorou a transferência de calor.

\section{Conclusão}

Referente ao estudo realizado, pode-se chegar ás seguintes conclusões:

Com a convecção forçada ocorre um aumento do coeficiente de convecção e portanto diminuição do $\Delta \mathrm{T}$ entre a superfície e o ambiente. $\mathrm{O}$ mesmo ocorre com o aumento da área superficial de transferência de calor. No novo projeto de aletas, com o aumento da área superficial ocorreu uma redução no $\boldsymbol{\Delta} T$, isso ocorreu também na convecção forçada.

Os valores encontrados para a eficiência da aleta foram todos próximos, devido às pequenas faixas de temperaturas que ocorrem no experimento e, consequentemente, aos baixos valores de coeficiente de convecção.

As imagens obtidas pelo termovisor na convecção forçada apresentaram regiões mais refrigeradas devido ao uso da ventoinha, se comparada com a convecção natural.

O uso da ventoinha para redutores de baixa potência não é prática usual, devido ao seu rendimento ser muito baixo. A relação da melhoria que ela proporciona para o sistema de convecção é irrelevante, comparada à energia que ela consome do eixo.

Com relação a trabalhos futuros, sugere-se:

Realização do experimento para redutores com maior potência nominal relativa, sendo realizados em bancadas de testes ou Software.

Análise para fabricação do redutor em alumínio injetado, utilizando matrizes no processo de fabricação, com a utilização deste processo poderia ser feito maiores alterações na geometria do redutor e na quantidade de aletas, aumentando a área superficial e o coeficiente de convecção, e melhorando a rugosidade. 


\section{Referências}

BRANDI, A. C. Desenvolvimento de uma técnica não intrusiva de medição do coeficiente de convecção: solução do problema térmico inverso. 2010. Tese (Doutorado em Térmica e Fluidos) Escola de Engenharia de São Carlos, Universidade de São Paulo, São Carlos.

CORRADI, C. A. L. Metalurgia das ligas de alumínio e tratamentos no metal líquido. Belo Horizonte: SENAI, 2001.

CREDER, H. Instalações elétrica. 15. ed. Rio de Janeiro: LTC, 2007.

HUANG, L. J.; SHAH, R. K. Assessment of calculation methods for efficiency of straight fins of rectangular profile. International Journal of Heat and Fluid Flow. v. 13, n. 3, p. 282-293, sept. 1992.

INCROPERA, F. P.; DEWITT, D. P. Fundamentos de transferência de calor e de massa. 6. ed. Rio de Janeiro: LTC, 2003.

KERN, D. Q. Procesos de tranferencia de calor. México: Compañia Editoral Continental, 1979.

KREITH, F.; BOHN, M. S. Princípios de transferência de calor. 6. ed. São Paulo: Pioneira Thomson Learning, 2003.

MELCONIAN, S. Elementos de máquinas. 9. ed. São Paulo: Érica, 2008.

MOBIL OIL. Catálogo técnico: Mobil Oil do Brasil Ind. Com. Ltda. Rio de Janeiro, 1999.

PANESI, A. R. Q. Fundamentos de eficiência energética. São Paulo: Ensino Profissional, 2006.

PIERINI. Catálogo técnico: Pierini Redutores. Criciúma, 2007.

SILVEIRA, F. R., CORREIA, W. B. Freio magnético para teste de rendimento de motores: aspectos de implementação e controle de conversor estático de potência. In: ENCONTROS UNIVERSITÁRIOS 2011, Fortaleza. Anais... Fortaleza, 2011.
WEG. Catálogo técnico: Weg. Jaraguá do Sul, 2012.

WELTY, J. R., WICKS, C. E., WILSON, R. E., RORRER, G. Fundamentals of momentum, heat, and mass transfer. 4 ed. Springfield, VA, USA. John Wily \& Sons Inc., 2001.

Recebido em 17 Setembro 2013- Received on September 17, 2013.

Aceito em 15 Março, 2014 - Accepted on March 15, 2014. 